\title{
Reactivity Improvement of Dicalcium Phosphate Dihydrate with Fluoride for Its Removal from Waste and Drinking Water
}

\author{
Yuka Takemura ${ }^{1}$, Masanori Kikuchi ${ }^{1,2}$, Masamoto Tafu ${ }^{3, *}$, Takeshi Toshima ${ }^{3}$, Tetsuji Chohji ${ }^{4}$ \\ ${ }^{1}$ Graduate School of Information Science and Technology, Hokkaido University, Japan \\ ${ }^{2}$ International Center for Materials Nanoarchitectonics (MANA), National Institute for Materials Science, Japan \\ ${ }^{3}$ National Institute of Technology, Toyama College, Japan \\ ${ }^{4}$ National Institute of Technology, Kagoshima College, Japan
}

Copyright $\subseteq 2016$ by authors, all rights reserved. Authors agree that this article remains permanently open access under the terms of the Creative Commons Attribution License 4.0 International License

\begin{abstract}
Dicalcium phosphate dihydrate (DCPD) reacts with fluoride ion in an aqueous solution and forms stable fluoroapatite (FAp). This reaction requires a lag time to form nano-sized precursor, hydroxyapatite (HAp)-like calcium phosphate, on DCPD surface. This long lag time prevents DCPD from applying as an effective fluoride removal agent from waste water. The purpose of this study is to improve the reactivity of DCPD with fluoride ion by HAp-coating on the DCPD surface by soaking it in the simulated body fluid (SBF) under various DCPD/SBF ratios and soaking periods. The results showed the HAp-coating on DCPD by soaking in the SBF reduced the lag time to be negligible with increasing in amount of HAp up to $2 \%$ in mass to the DCPD; however, further HAp formation had no affects on improvement of the reactivity.
\end{abstract}

Keywords Calcium Phosphate, Nano-Hybridization, Hydroxyapatite Coating, Water Treatment

\section{Introduction}

Calcium phosphates (CaPs) are widely used as food additives, fertilizers and biomaterials with variety of compounds in different ratios of calcium and phosphate. Low soluble $\mathrm{CaPs}$ gradually convert into a more stable $\mathrm{CaP}$ in an aqueous solution depended upon $\mathrm{pH}$, temperature and ions in the solution mainly according to the solubility of $\mathrm{CaP}$ [1]. Calcium phosphate with the lowest solubility in the range of weak acid $(\mathrm{pH} \approx 5)$ to weak basic $(\mathrm{pH} \approx 10)$ is a solid solution between hydroxyapatite (HAp, $\left.\mathrm{Ca}_{10}\left(\mathrm{PO}_{4}\right)_{6}(\mathrm{OH})_{2}\right)$ and fluoroapatite $\left(\mathrm{FAp}, \mathrm{Ca}_{10}\left(\mathrm{PO}_{4}\right)_{6} \mathrm{~F}_{2}\right)$ in which fluoride amount depends on fluoride ion concentration. In fact, FAp has the lowest solubility in all calcium phosphate in that $\mathrm{pH}$ range; thus, precipitation of $\mathrm{FAp}$ is widely used for immobilization of fluoride ion in the water environment, such as wastewater.

In general, fluoride ions in waste water is roughly removed as $\mathrm{CaF}_{2}$ by addition of calcium compounds to the water, and remaining low concentration fluoride is removed as FAp by mixing with calcium and phosphate compounds; however, the following problems are remained to achieve maximum efficiency of the water treatment: Control of calcium and phosphate amounts, fine-tuning of reaction conditions and particle size of FAp. Formation of small FAp particles obtained from aqueous solution at room temperature inhibit efficient separation of FAp slug and water [2].

We recently have been focusing on transforming reaction of comparatively high soluble calcium phosphates into FAp to solve the problems mentioned above, i.e., the calcium phosphates added to the waste water worked as a source of calcium and phosphate to immobilize fluoride ion as FAp and as a template of FAp formation to obtain its large particle for easy separation from water.

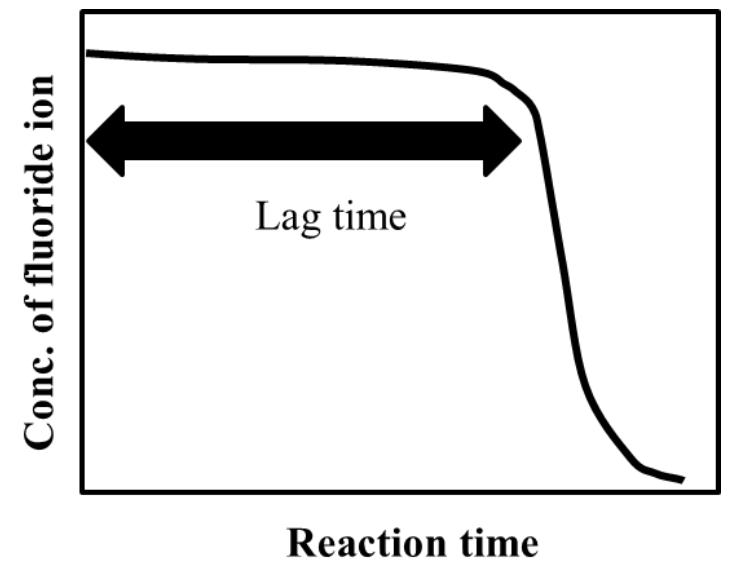

Figure 1. Change in the concentration of fluoride ion when addition of DCPD to F-containing water. 
Dicalcium phosphate dihydrate (DCPD, $\mathrm{CaHPO}_{4} \cdot 2 \mathrm{H}_{2} \mathrm{O}$ ) is a good candidate for fluoride ion removal from water because it reacts with fluoride ions in an aqueous solution and forms stable FAp [3][4]. We have been investigating this conversion reaction to apply DCPD to the removal process of fluoride ion in the water environments such as waste and drinking water [5][6][7][8], and found that DCPD did not react with fluoride ion directly but required previous formation of nano-sized precursor on the DCPD surface [7]. The precursor formation needed 1-3 $\mathrm{h}$ or even more leading time that we called as "lag time" as shown in Figure 1. The lag time is one of the big problems to apply DCPD to fluoride ion removal from water, because it leads long water treatment period. In our previous studies, the nano-sized precursor is HAp-like calcium phosphate, and addition of HAp particles in DCPD improves the reactivity of fluoride ion with DCPD but the templating effect of DCOD was missing [9]. These result suggested that pre-formation of HAp particles on DCPD particles could be a simple and good method to improve the reactivity with fluoride ion to solve the lag time problem without missing of the templating effect. In the biomaterial field, simulated body fluid (SBF) is widely used to form nano-sized HAp coating on ceramics, metal and polymers [10]; therefore, HAp nanoparticles coating on the surface of DCPD could be realized with the SBF. The formation of HAp on the bioactive surface in the SBF is generally due to supplying of $\mathrm{Ca}$ from materials as well as functional groups for HAp nucleation, such as $\mathrm{Si}-\mathrm{OH}$ in bioactive glass and silica gel. We hypothesized that supplementation of $\mathrm{Ca}$ from DCPD could occur by surface dissolution, and $\mathrm{HPO}_{4}$ groups on the DCPD surface could be a candidate of functional group for HAp nucleation on the DCPD surface could be $\mathrm{HPO}_{4}$ group. Further, DCPD changes very slowly into HAp at the SBF condition by dissolution-precipitation process.

In the present study, we investigated suitable conditions of HAp-coating by the SBF soaking method [11] and improvement of the reactivity of DCPD with fluoride ion by fluoride ion uptake from water with the DCPD.

\section{Materials and Methods}

\subsection{Materials}

Reagent grade of DCPD (Taihei Chemical, Japan) was used in this study. Water used in this study was prepared by an ion exchange and ultra-purification using Milli-Q system (Merck Millipore, Germany). Fluoride solution was prepared by diluting $1000 \mathrm{mg} / \mathrm{L}$ fluoride standard solution (sodium fluoride, Wako Pure Chemical, Japan) with the water. The simulated body fluid (SBF), chosen for coating of HAp on the DCPD surface [11], was prepared according to the protocol described by $\mathrm{Cho}$ et al. using $\mathrm{NaCl}$, $\mathrm{NaHCO}_{3}, \mathrm{KCl}, \mathrm{K}_{2} \mathrm{HPO}_{4} \cdot 3 \mathrm{H}_{2} \mathrm{O}, \mathrm{MgCl}_{2} \cdot 6 \mathrm{H}_{2} \mathrm{O}, \mathrm{CaCl}_{2}$, $\mathrm{Na}_{2} \mathrm{SO}_{4}$ and $\left(\mathrm{CH}_{2} \mathrm{OH}\right)_{3} \mathrm{CNH}_{2}$ (Nakalai Tesque, Japan) [12].

\subsection{Experimental Procedure}

\subsubsection{Coating of HAp on DCPD}

One gram of the DCPD was added in $10,20,30$ or $50 \mathrm{~mL}$ of SBF in a polycarbonate bottle. The suspension was shaken with the reciprocal shaker (Type MMS-3010, EYELA, Japan) for 1,3 or 7 days at $25^{\circ} \mathrm{C}$ with an incubator (Type FMC-1000, EYELA, Japan). After the treatment, the solid phase in the suspension was collected by a vacuum filtration with a 0.45 $\mu \mathrm{m}$ membrane filter (OMNIPORE MEMBRANE FILTERS, Merck Millipore, Germany) and dried at $40^{\circ} \mathrm{C}$ in a drying oven. Surface of the solid phase was observed with a scanning electron microscope (SEM, JSM-6390AX, JEOL, Japan), and its crystal phase was identified with a powder X-ray diffractometer (XRD, Miniflex ${ }^{\circledR}$, Rigaku, Japan). One milligram of solid phase was dissolved in $10 \mathrm{~mL}$ of $1 \mathrm{~mol} / \mathrm{L}$ $\mathrm{HNO}_{3}$ solution and served for quantitative analysis of $\mathrm{Ca}$ and $\mathrm{P}$ with an inductively coupled plasma atomic emission spectrometer (ICP-AES, Type 720-ES, Varian-Agilent, USA) to determine its $\mathrm{Ca} / \mathrm{P}$ atomic ratio. Mass ratio of HAp and DCPD was also estimated from the $\mathrm{Ca} / \mathrm{P}$ ratio with a hypothesis that $\mathrm{Ca} / \mathrm{P}$ ratio of HAp and DCPD were stoichiometric ones, 1.67 and 1.00, respectively.

\subsubsection{Evaluation of reactivity of DCPD with Fluoride Ion}

Twenty milligrams of the raw DCPD or the HAp-coated DCPD was added into the $20 \mathrm{~mL}$ of $20 \mathrm{mg} / \mathrm{L}$ fluoride solution. The suspension was shaken with the reciprocal shaker at $25^{\circ} \mathrm{C}$ for 1,3 or 6 hours. After the shaking, the suspension was separated by a pressure filtration with the $0.45 \mu \mathrm{m}$ membrane filter. Fluoride concentration of the filtrate mixed with total Total ionic strength adjustment buffer (TISAB II, METTLER TOLEDO, Switzerland) at 10:1 volume ratio was measured of $1 \mathrm{~mL}$ was added to 10 $\mathrm{mL}$ of the filtrate, and its fluoride concentration was measured with a $\mathrm{pH} / \mathrm{ion}$ meter (Type Seven multi-S50, METTLER TOLEDO, Switzerland) equipped with a fluoride ion selective electrode (ISE, Type Orion 9609BNWP, Thermo Electron, USA). A reactivity with fluoride ions was evaluated by comparing fluoride concentrations before and after treatment with the DCPD treatment.

\section{Results and Discussions}

\subsection{Preparation of HAp-coated DCPD}

Figure 2 shows powder X-ray diffraction patterns of the solid phase before and after soaking in various volumes of the SBF for 1 day. Diffraction peaks of HAp and DCPD were detected fro $\mathrm{Ca} / \mathrm{P}$ ratios, were summarized in Table 1 . Amount of HAp was increased with increasing in both the volume of SBF, as estimated from XRD data, and soaking time. Thus, amounts of HAp formed by the SBF soaking were controlled by the volume of SBF and soaking time. 


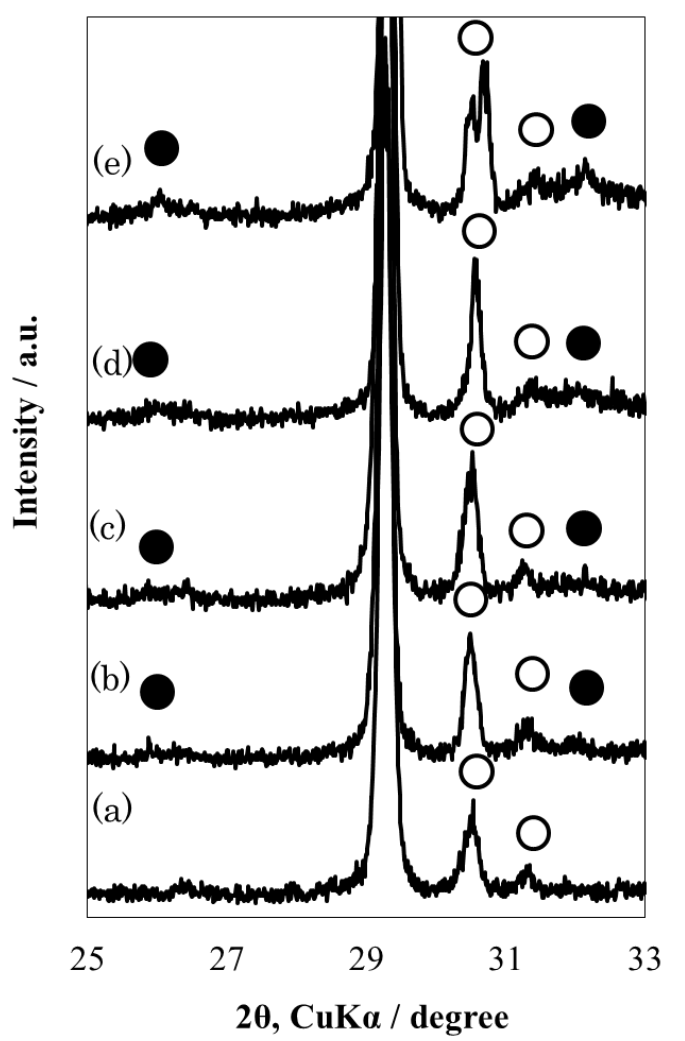

Figure 2. Powder X-ray diffraction patterns of raw DCPD and DCPDs soaked with various volume of SBF for 1 day. (a) : raw DCPD, DCPDs soaked in (b) : $10 \mathrm{~mL}$, (c) $: 20 \mathrm{~mL}$, (d) $: 30 \mathrm{~mL}$, and (e) $: 50 \mathrm{~mL}$ of SBF. Open and closed circles indicated peaks from DCPD and HAp phases respectively.

Table 1. Amount of HAp coated on DCPD estimated from $\mathrm{Ca} / \mathrm{P}$ atomic ratio.

\begin{tabular}{|c|c|c|c|}
\hline \multirow{2}{*}{$\begin{array}{c}\text { SBF } \\
\text { Soaking time } \\
\text { amount } / \mathrm{mL}\end{array}$} & \multicolumn{3}{|c|}{$\begin{array}{c}\text { Amount of HAp / \% } \\
\text { in mass }\end{array}$} \\
\hline & 1 & 3 & 7 \\
\hline 10 & 0.49 & 1.8 & 12 \\
\hline 20 & 3.5 & 7.6 & 14 \\
\hline 30 & 3.7 & 5.4 & 14 \\
\hline 50 & 6.4 & 8.7 & 19 \\
\hline
\end{tabular}

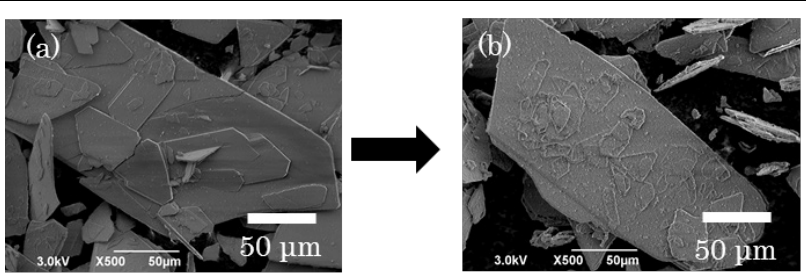

(1) Shape of DCPD

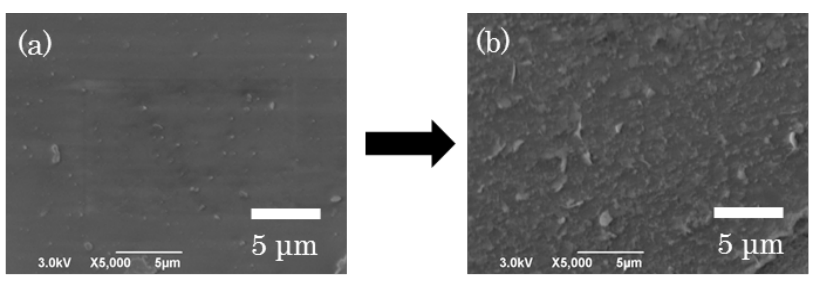

(2) Surface of DCPD

Figure 3. Changes of DCPD by soaking SBF in $50 \mathrm{~mL}$ for 1 day. Raw DCPD (a) and DCPD after soaking in SBF (b).

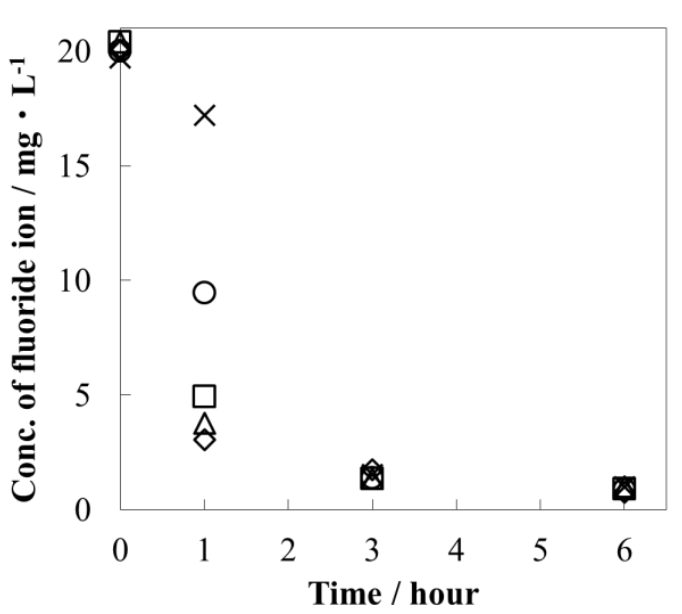

Figure 4. Time dependent changes in fluoride ion concentrations of fluoride ion solution after treated with DCPDs, raw DCPD (cross) and DCPDs coated with HAp by 1 day soaking in SBF amount of $10 \mathrm{~mL}$ (open circle), $20 \mathrm{~mL}$ (open square), $30 \mathrm{~mL}$ (open triangle) and $50 \mathrm{~mL}$ (open diamond).

Figure 3 shows SEM photographs of the DCPD before and after soaking in $50 \mathrm{~mL}$ of the SBF for 1 day. The particle shape of the DCPD maintained even after soaking; however, formation of small particles on the surface of DCPD was observed as shown in the Fig. 3 (2), and particles could be HAp precipitated from the SBF by the dissolution-precipitation process as detected discussed in powder XRD. Similar results were also obtained in other treatment conditions.

Therefore, HAp was successfully coated on surface of the DCPD particles and its amount was controlled by soaking conditions, time and volume, in the SBF.

\subsection{Influence of HAp Coating on Reactivity with Fluoride Ion}

Figure 4 shows the reactivity with fluoride ions of the DCPDs coated with HAp at various volumes of SBF for 1 day. Although differences in fluoride ion concentrations among these HAp-coated DCPDs were observed at $1 \mathrm{~h}$, no differences were observed at 3 and $6 \mathrm{~h}$. The fluoride ion concentration after treatment with the raw DCPD was higher than that with the HAp-coated DCPDs; moreover, the concentration was decreased with increasing in volume of SBF in which the DCPD soaked. This result suggested that HAp-coating DCPD could reduce the lag time. The similar results were obtained from other SBF soaking time.

\subsection{Optimum condition of HAp-coating}

In our previous studies, addition of HAp particles in DCPD improves the reactivity of fluoride ion with DCPD to shorter the lag time less than $1 \mathrm{~h}$ [9]. Thus, differences of fluoride ion concentration at $1 \mathrm{~h}$ might reflect the lag time, and could be used as an index of lag time existence, i.e., an index of improvement in reactivity. 
Figure 5 shows the relation between the amount of HAp coating and fluoride ion concentration after $1 \mathrm{~h}$ treatment with DCPDs. Fluoride ion concentration depended upon the amount of HAp coating, but not depended upon SBF soaking time nor SBF amount. The fluoride ion concentration reached plateau at $2 \%$ in mass of the HAp coating; thus the optimum amount of HAp coating was determined as $2 \%$ in mass to the DCPD. This amount was drastically smaller than that of the HAp mixing method, $30 \%$ in mass [9]. In case of the HAp mixing, although increasing in the amount of HAp shortens the time to start reaction with fluoride ions, the reaction amount of fluoride ions decreases [13]. Contrarily in case of the HAp-coating, reaction amount of fluoride ions did not change even the amount of HAp became larger than $2 \%$. Therefore, it can be expected that the HAp coating can reduce the amount of treatment agent including DCPD in water treatment in comparison to the HAp mixing.

Further, FAp formed from the HAp-coated DCPD maintained initial DCPD shapes [9] as the same as that formed from the DCPD as in our previous study [7], while that from the HAp-mixed DCPD has quite smaller size than that from the DCPD [9]. Particle size of FAp formed from the DCPD is not change significantly from it of initial DCPD [14]. These difference came from crystals for template of FAp formation. The DCPD crystal was worked as a template with formation of precursor, apatite-like calcium phosphate, on its surface; however, HAp mixed with DCPD was preferable for epitaxial growth of FAp due to quite smaller mismatch between the FAp and HAp crystal lattices than that between FAp and DCPD. Contrarily, HAp coating on the DCPD simulated precursor formation; thus, templating effect of the DCPD remained. These results suggested that FAp particle size from the HAp-coated DCPD could be controlled by initial DCPD particle sizes. In general, separation of sludge containing small particles from the suspension requires time and effort due to clogging of filter by these small particles. Formation of large FAp particles from the HAp-coated DCPD with appropriate size could improve a separation of the obtained sludge composed of large particle of FAp as well as the reactivity with fluoride ions.

\section{Conclusions}

The reactivity of DCPD with fluoride ions was improved by the DCPD coated with HAp by soaking in the SBF. The SBF soaking formed the HAp layer on the DCPD by a dissolution-precipitation process, and the HAp amount can be controlled by the SBF amount and soaking time. The reactivity with fluoride ions depended on the amount of HAp coating; however, the reactivity reached plateau at $2 \%$ in mass of HAp coating. Therefore, the optimum amount of HAp for coating was determined as $2 \%$ in mass to the DCPD that is quite small in comparison to the HAp amount for the HAp-mixing method, $30 \%$ in mass. Further, the
HAp-coated DCPD could from large size FAp particles,

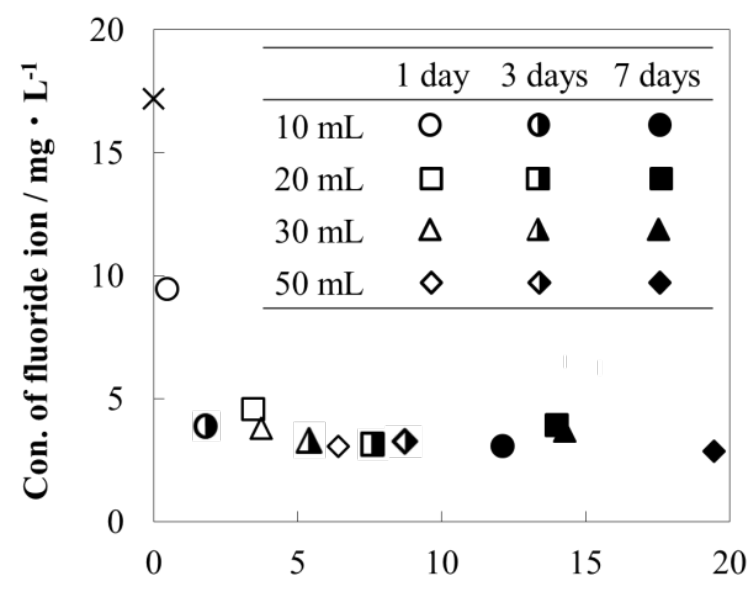

The amount of HAp coating / \%

Figure 5. Relations between amount of HAp coating and concentration of fluoride ion at $1 \mathrm{~h}$ after treatment with HAp-coated DCPDs prepared with various conditions shown in the inlet table.

which would improve the separation of sludge from suspension after treatment as well.

These results suggested that the DCPD coated with HAp by soaking in the SBF was quite effective to shorten the reaction time with fluoride ions as well as sludge removal time from the suspension after treatment. The HAp-coated DCPD is expected to be a good candidate for fluoride ion removal in a water treatment system.

\section{Acknowledgements}

The authors wish to thank for generous supports from Dr. Takumi Fujita and his stuff in Chiyoda Ute Co. Ltd., Japan.

A part of this study was supported by the KAKENHI Grant-in-Aid for Scientific Research (B) (23310058) from the Japanese Society for the Promotion of Science (JSPS).

\section{REFERENCES}

[1] Koutsoukos, P. G., Current Knowledge of Calcium Phosphate Chemistry and in Particular Solid Surface-Water Interface Interactions, In Proceedings $2^{\text {nd }}$ International Conference on the Recovery of Phosphorus from Sewage and Animal Wastes, Noordwijkerhout, The Netherlands, 18pp, 2001

[2] Mori, M., Watanabe, S., Wakayama, H., Ando, J., Removal of Fluorine from Waste-Waters (II) Removal in a Form of Fluorapatite, Gypsum \& Lime, Vol.1976, No. 140, 14-16, 1976

[3] Duff, E.J, Orthophosphate-I. Formation of Apatites from Calcium Phosphates in Potassium Fluoride Solutions, J. Inorg. Nucl. Chem., Vol.32, 3103-3106, 1970

[4] Duff, E.J, Orthophosphates. Part II. The transformations 
brushite $\rightarrow$ fluoroapatite and monetite $\rightarrow$ fluoroapatite in aqueous potassium fluoride solution, J. Chem. Soc. A., Vol.1971, 33-38, 1971

[5] Tafu, M., Chohji, T., Korenaga, T., Treatment of Fluoride in Wastewater and Waste Gypsum Board Based on Nano-Surface Reaction of Calcium Phosphate, J. Envi. And Safety, Vol.1, No.2, 33-39, 2010

[6] Tafu, M., Nagamori, T., Chujo, Y., Toshima, T., Morioka, I., Nakano, H., Fujita, T., Application of calcium phosphate dihydrate (DCPD) to wastewater treatment, Arch. Bioceram. Res., Vol.9, 191-194, 2009

[7] Tafu, M., Chohji,T., Reaction of Calcium Hydrogenphosphate Dihydrate (DCPD) with a solution containing a small amount of fluoride, J. Ceram. Soc. Jpn, Vol.113, 363-367, 2005

[8] Tafu, M., Chohji, T., Morioka, I., Hiwasa, M., Nakano, H., Fujita, T., Stabilization of Fluoride in Waste Gypsum by Using Surface-Modified Calcium Phosphate Particle, Trans. MRS-J, Vol.35, No.2, 377-380, 2010

[9] Tafu, M., Masutani, T., Takemura, Y., Toshima,T., Chohji,T.,
Effect of Hydroxyapatite on Reaction of Dicalcium Phosphate Dihydrate (DCPD) and Fluoride Ion, Bioceram. Dev. Appl., S1-15, 2013

[10] Kokubo. T., Takadama. H., How useful is SBF in predicting in vivo bone bioactivity?, Biomaterials, Vol.27, No.15, 2907-2915, 2006

[11] Tas, A. C., Bhaduri, S. B., Chemical Processing of $\mathrm{CaHPO}_{4} \cdot 2 \mathrm{H}_{2} \mathrm{O}$ : Its Conversion to Hydroxyapatite, J. Am. Ceram. Soc., Vol.87, 2195-2200, 2004

[12] Cho, S.B., Nakanishi, K., Kokubo, T., Soga, N., Ohtsuki, C., Nakamura, T., Yamamuro, T., Dependence of Apatite Formation on Silica gel on Its Structure: Effect of Heat Treatment, J. Am. Ceram. Soc., Vol.78, 1769-1774, 1995

[13] Tafu, M., Chohji, T., Toshima, T., Fujita, T., Nakano, H., Morioka. I., Mori, K., Maeda, M., Takenaka, K., Fluorine insolubilizers and methods of producing same, United States Patent, 8333908, 2012

[14] Tafu, M., Arioka, Y., Takamatsu, S., Toshima, T., Properties of sludge generated by the treatment of fluoride-containing wastewater with dicalcium phosphate dihydrate, Euro-Mediter. J. Environ. Integ., Submitted 